\title{
ATMOSPHERIC CORRECTION COMPARISON OF SPOT-5 IMAGE BASED ON MODEL FLAASH AND MODEL QUAC
}

\author{
Yunkai GUO ${ }^{\text {a }}$, Fan ZENG ${ }^{\text {a }}$ \\ ${ }^{\text {a }}$ Changsha University of Science \& Technology, School of Traffic \& Transportation Engineering \\ Changsha, Hunan, China, 410004, guoyunkai226@163.com
}

KEY WORDS: Atmosphere, Comparison, Retrieval, Model, SPOT-5, RVI, Spectral

\begin{abstract}
:
Atmospheric correction of satellite remote sensing image is the precondition of quantitative remote sensing study, and also among the difficulties of it. There are various methods and models for atmospheric correction. The author makes the atmospheric correction of SPOT-5 multi-spectrum remote sensing image covering Changsha, Zhuzhou and Xiangtan by adopting Model FLAASH and Model QUAC in the trail, and then makes a contrastive analysis of the image before and after the correction from the point of sight, surface features spectral curve and RVI result. The results show that both models with their specific scope of application can both basically eliminate the atmospheric effects and can restore the typical characteristics of various surface features spectral better, emphasis the vegetation information; the one using Model FLASSH has higher accuracy than the one using Model QUAC; it is more convenient to use Model QUAL than Model FLASSH, because it has little dependence on input parameters and calibration accuracy of instruments.
\end{abstract}

\section{INTRODUCTION}

Electromagnetic waves need to pass through the atmosphere before being received by the sensor, in which process the atmosphere would absorb and scatter the sunshine and radiation from the targets, so the original remote sensing image includes both the surface information of physical body and the information of the sun and the atmosphere, and the correction process of eliminating these atmospheric effects is called atmospheric correction (c: Chen Shupeng etc, Study of Information Mechanism of Remote Sensing). With the increasing development of remote sensing technique, atmospheric correction of remote sensing images requires a gradual increase, and the research on its methods are being paid more and more attention (c: Zhao Yingshi etc, Applications of Remote Sensing Principles and Methods). The atmospheric correction of remote sensing images began in 1970s, and after years of development, the methods for atmospheric correction can be broadly divided into three kinds: the method based on radioactive transfer model, the method of relative correction based on image characteristics and the method based on ground linear regression model. Among them, the method based on radioactive transfer model is more used in satellite images with high precision of the calculated reflectivity, but it is vulnerable to the impact of access to real-time atmospheric parameters; the method of relative correction based on image features is to eliminate atmospheric effect directly of the image features itself, but it needs some known or assumed values of the reflectivity of the pixel; the key of the method based on ground linear regression model is to establish the linear regression equation between the ground target and the corresponding pixel of remote sensing image, and the advantage of this method is that the physical meaning is clear and the calculation is simple, the disadvantage is that it depends more on the field work with high cost (c: Yang Jiaojun etc, Effect on Atmospheric Correction by Inputting Parameters of Model).

FLASSH based on atmospheric radioactive transfer model is commonly used among the atmospheric correction methods at present. It can make atmospheric correction on the hyper spectral and multispectral data, the atmospheric attribute properties inverted pixel by pixel, but it depends on the input atmospheric parameters and calibration precision of instruments. Model QUAC depends less on the atmospheric parameters, relatively easy to achieve, and it also has its specific application scope although its calibration accuracy is not as high as Model FLAASH. Many researchers have made atmospheric correction study on various images by using different methods of atmospheric correction, such as M.W. Matthew, S. M. Adler-Golden, who make atmospheric correction research on AVIRIS data by using Model FLAASH, Song Xiaoyu, Wu Bin, who evaluate Model FLAASH by using AVIRIS, Hyperion and other high spectral data, B.-C.Gao, M. J. Montes, who carry on research on rapid atmospheric correction algorithm based on hyperspectral remote sensing data, Yang Hang make a comparison between FLAASH and empirical line method on their application of OMIS- II image atmospheric correction, etc, all these studies have achieved an ideal result. Nevertheless, the comparative study on atmospheric correction of SPOT-5 image by using Model FLAASH and Model QUAC is quite few at present. This paper mainly discusses the atmospheric correction of SPOT-5 image by using Model FLAASH and Model QUAC, and makes a contrastive analysis of the image in the aspects of sight and surface features spectral curve to obtain the actual correction effects of these two models.

\section{DATA SOURCE AND STUDY AREA}

Remote sensing data selected for research is SPOT-5 1A data after the first class radiation correction, and the spatial resolutions of its multi-spectral and panchromatic image are separately 10 meters and 5 meters. Study area is within Changsha, Zhuzhou and Xiangtan which are typically hilly areas in the southern China, and also includes the city and the surrounding areas. Date of data acquisition is November 2, 2010, and due to the cloudless day, the data is of high quality and its band and wavelength range are shown in Table 1. In order to verify the effect of atmospheric correction better, this 
study used wild spectroradiometer ISI921VF for wild spectral measurements; the band range, which is fitted within the band range of SPOT-5 satellite through spectral resampling, is 380 1050nm of visible light and near infrared light.

\begin{tabular}{|c|c|}
\hline Band & Wavelength Range(um) \\
\hline Panchromatic & $0.48 \sim 0.71$ \\
B1(Green) & $0.50 \sim 0.59$ \\
B2(Red) & $0.61 \sim 0.68$ \\
B3(Near infrared) & $0.78 \sim 0.89$ \\
B4(Short wave infrared) & $1.58 \sim 1.75$ \\
\hline
\end{tabular}

Table 1. Bands and wavelength range of SPOT-5 satellite

Before the atmospheric correction, first make the geometric correction on the SPOT-5 image by using the coordinate data of the known control point, and then transform the DN value of SPOT-5 into radiance (Unit: $\mu \mathrm{w} \cdot \mathrm{cm}^{-2} \cdot \mathrm{nm}^{-1} \cdot \mathrm{sr}^{-1}$ ) and apparent reflectance. The transformation process can be finished through the Modeler of ERDAS IMAGINE9.2.

\section{ANALYSIS OF APPLICATION AND EFFECT OF ATMOSPHERIC CORRECTION}

\subsection{Model FLAASH atmospheric correction}

FLASSH is developed by both Spectral Science, Inc. (SSI), the leader of the research on atmospheric correction algorithm, and Air Force Research Labs(AFRL). It combines radioactive transfer code of MODTRAN4+ and has been modified on this basis. FLAASH is the tool for first-principle atmospheric correction, which is able to correct from the visible light, near infrared light to shortwave infrared light and can also eliminate most of the influence which the air and light and other factors have on clutter reflectance to obtain more accurate parameters of reflectivity, emissivity, surface temperature and other real physical models of surface features.

FLAASH begins with a standard equation of spectral radiance of the single pixel received by a standard planar lambertian (or nearly a planar lambertian), which is based on the sun spectrum (not including thermal radiation), by the sensor.

$$
L=\left(\frac{A \rho}{1-\rho_{e} S}\right)+\left(\frac{B \rho_{e}}{1-\rho_{e} S}\right)+L_{a}
$$

Thereinto, L' is the radiance for the single pixel received by the sensor; $\rho$ is the surface reflectance for the pixel; $\rho_{\mathrm{e}}$ is the average surface reflectance for this pixel and surrounding pixels; $\mathrm{S}$ is the spherical albedo for the atmosphere; $\mathrm{L}_{\mathrm{a}}$ ' is the radiance when atmosphere radiation enters into the sensor, A,B is the coefficient determined by the atmospheric conditions and the geometric conditions of underlying surface with nothing to do with surface reflectance.

(A $\rho /\left(1-\rho_{\text {e }} \mathrm{S}\right)$ ) indicates the radiation energy entering into the sensor directly from the target surface features, which implies two cases: the reflection happening when the sun irradiates on the target surface features; the neighboring surface features scatter through the atmosphere and then irradiate on the surface features for reflection again ${ }^{[4]}$. (B $\rho_{e} /\left(1-\rho_{e} S\right)$ ) indicates the amount of radiation which enters into the sensor through the atmosphere from the surface. The difference between $\rho$ and $\rho_{\text {e }}$ explains "proximity effect" (mixed radiation close to pixel) caused by the atmospheric scattering, in order to ignore this proximity effect, so $\rho=\rho$ e. However, there will be significant errors when there is mist or a strong contrast between the surfaces.

According to the Formula (1), surface reflectance can be calculated pixel by pixel. FLAASH uses spatial average radiancy, ignoring the "proximity effect", to get approximate equation (2) and to estimate the spatial average reflectance. Thereinto, $L_{e}$ is the spatial average radiation image generated by convolution with the radiation image and spatial weighting function.

$$
L_{e} \approx\left(\frac{(A+B) \rho_{e}}{1-\rho_{e} S}\right)+L_{a}
$$

Most of the atmospheric correction parameters used in this trial are from the header file of image data, and the specific parameter data is shown in Table 2. After obtaining the required parameter, the true surface reflectance of the whole image can be calculated pixel by pixel by using Equation 1 and Equation 2 .

\begin{tabular}{|cccc|}
\hline $\begin{array}{c}\text { Date of } \\
\text { Imaging }\end{array}$ & $\begin{array}{c}\text { Time of } \\
\text { Imaging }\end{array}$ & Sensor & $\begin{array}{c}\text { Height of } \\
\text { Sensor }\end{array}$ \\
\hline 2010.11 .2 & $3: 28: 14$ & SPOT-5 & $800 \mathrm{~km}$ \\
\hline
\end{tabular}

\begin{tabular}{|cccc|}
\hline $\begin{array}{c}\text { Atmospheric } \\
\text { Model }\end{array}$ & $\begin{array}{c}\text { Aerosol } \\
\text { Type }\end{array}$ & $\begin{array}{c}\text { Center } \\
\text { Longitude } /\left({ }^{\circ}\right)\end{array}$ & $\left.\begin{array}{c}\text { Center } \\
\text { Latitude } /\left({ }^{\circ}\right.\end{array}\right)$ \\
\hline MLW & Rural & 113.1001 & 28.0001 \\
\hline
\end{tabular}

\begin{tabular}{|cccc|}
\hline $\begin{array}{c}\text { Elevation Angle of } \\
\left.\text { the Sun } /{ }^{\circ}\right)\end{array}$ & $\begin{array}{c}\text { Azimuth of the } \\
\left.\text { Sun } /{ }^{\circ}\right)\end{array}$ & Altitude & Visibility \\
\hline 46.192779 & 164.892501 & $0.037 \mathrm{~km}$ & $35 \mathrm{~km}$ \\
\hline
\end{tabular}

Note: Imaging time is GMT with a difference of 8 hours between GMT and Beijing Time.

Table 2. Input parameters of FLAASH atmospheric correction

\subsection{Model QUAC atmospheric correction}

Model QUAC is the method of atmospheric correction for hyper spectral and multispectral images from the visible light, near infrared light to shout-wave infrared light. QUAC is different from the first-principle atmospheric correction, because it obtains atmospheric compensating parameters directly from the image (observe pixel spectral) without complete information to achieve the atmospheric correction more approximate than FLAASH.

$$
\rho^{\prime}=\left(\rho_{1}+\rho_{2}+\cdots+\rho_{n}\right) / n
$$

As shown in Equation (3) below, QUAC is based on experience to collect the average reflectance of different substances, such as the end member spectrum in the field of vision. $\mathrm{n}$ indicates the number of end members, essentially independent field of vision, which means a faster operating rate than the 
first-principle method. Its model principle process is shown in Figure 1(c: ITT Visual Information Solutions (ITT VIS), “ENVI User’s Guide, Version 4.8”).

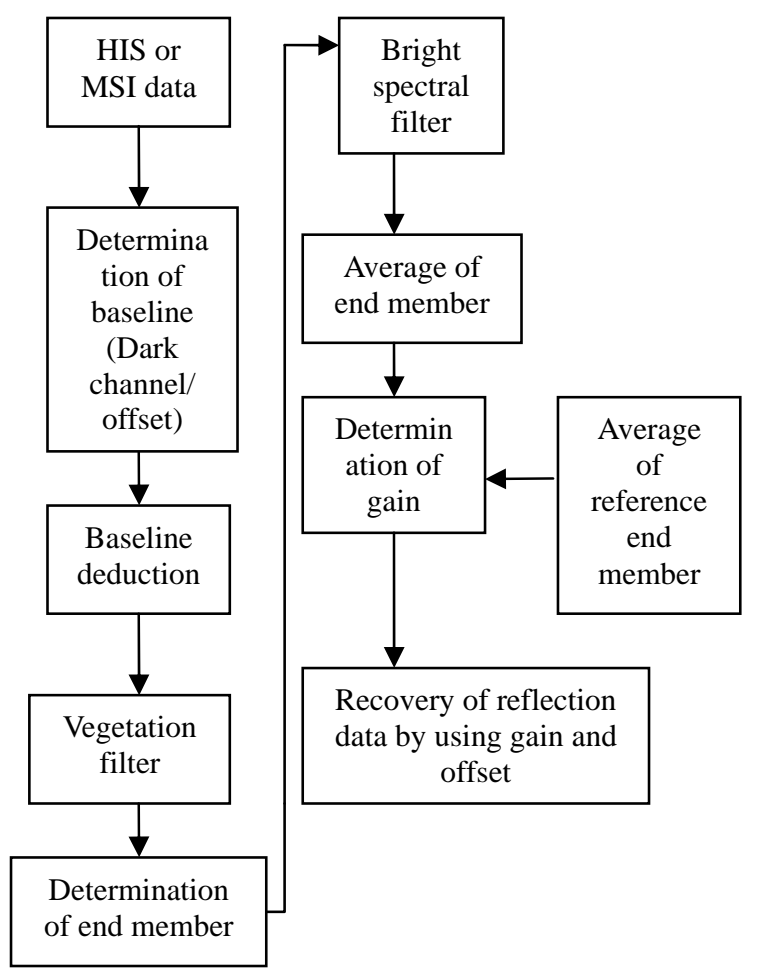

Figure 1. Flow chart of QUAC model

QUAC also needs the elevation angle of the sun and center wavelength. If the sensor has no correct radiation or wavelength calibration, or the sun light intensity (when there is cloud deck) is unknown, correction can still be made with this method within the allowed accuracy scope.

\subsection{Analysis of effect of atmospheric correction}

In order to evaluate and verify the effects of atmospheric correction which Model FLAASH and Model QUAC have on SPOT-5 remote sensing image, a contrastive analysis shall be made respectively for the images of both models after and before the correction in the aspect of sight and spectral curve of surface features reflectance. The images (geometric correction has been finished) before and after the atmospheric correction need geographical link first to ensure that the images of various scenes correspond to the same pixel in the same area when making the contrastive analysis.

3.3.1 Analysis of visual contrast before and after correction: In Figure 2, a, b, c is respectively the image in the same region after twice magnification before the atmospheric correction, after the FLAASH atmospheric correction and the QUAC atmospheric correction, and band RGB combination is 4, 3, 2 . We can see that there are obvious changes in visual effects of the image before and after the correction, darker for a on the whole, because the presence of the atmosphere will reduce the difference between light and dark of surface features to reduce contrast ratio of the images; visual effect of image after the correction has been improved significantly, brighter and clearer, contrast ratio also increased and image quality improved, indicating that atmospheric correction has effectively eliminated the effect of atmospheric aerosols, water vapor and other atmospheric factors. In contrast, image quality of $b$ (through FLAASH atmospheric correction) is slightly better than c (through QUAC atmospheric correction) due to more abundant information.

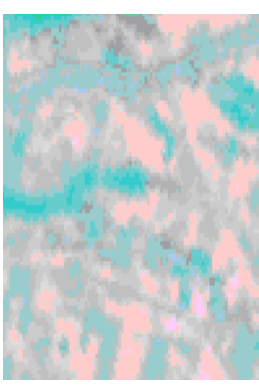

a

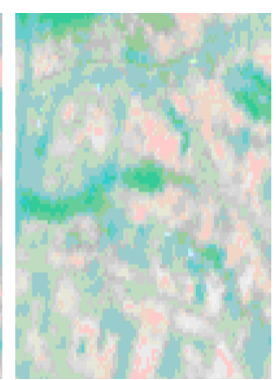

b

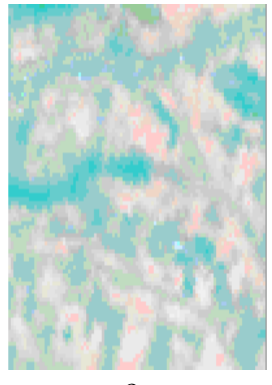

Figure 2. Visual analysis of atmospheric correction

3.3.2 Contrastive analysis of reflectance spectral curve: Characteristics of reflectance spectrum curve are an important means for the recognition of remote sensing image surface features. From the visual angle, only a rough evaluation can be made on the effects of the two atmospheric correction models, and making a contrast analysis of reflectance spectral curve of its corresponding typical surface features can reflect the effect of atmospheric correction better.

The study area of this trial is typically hilly area in southern China; data acquisition time is in November; the image presented mainly small ponds, the harvested farmlands and several scattered hills; and most of the surface is soil. Therefore, soil, vegetation, water body (pond water) and asphalt road are selected respectively as the surface type of pixel. As shown in Figure 3, a, b, c, d are respectively the figures of soil, vegetation, water bodies and asphalt road, four typical surface features, after FLAASH atmospheric correction, after QUAC atmospheric correction and of actual measurement reflectance curve.

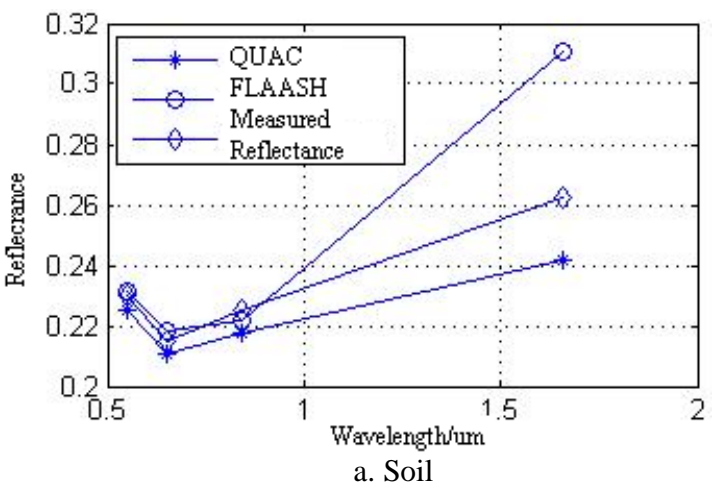



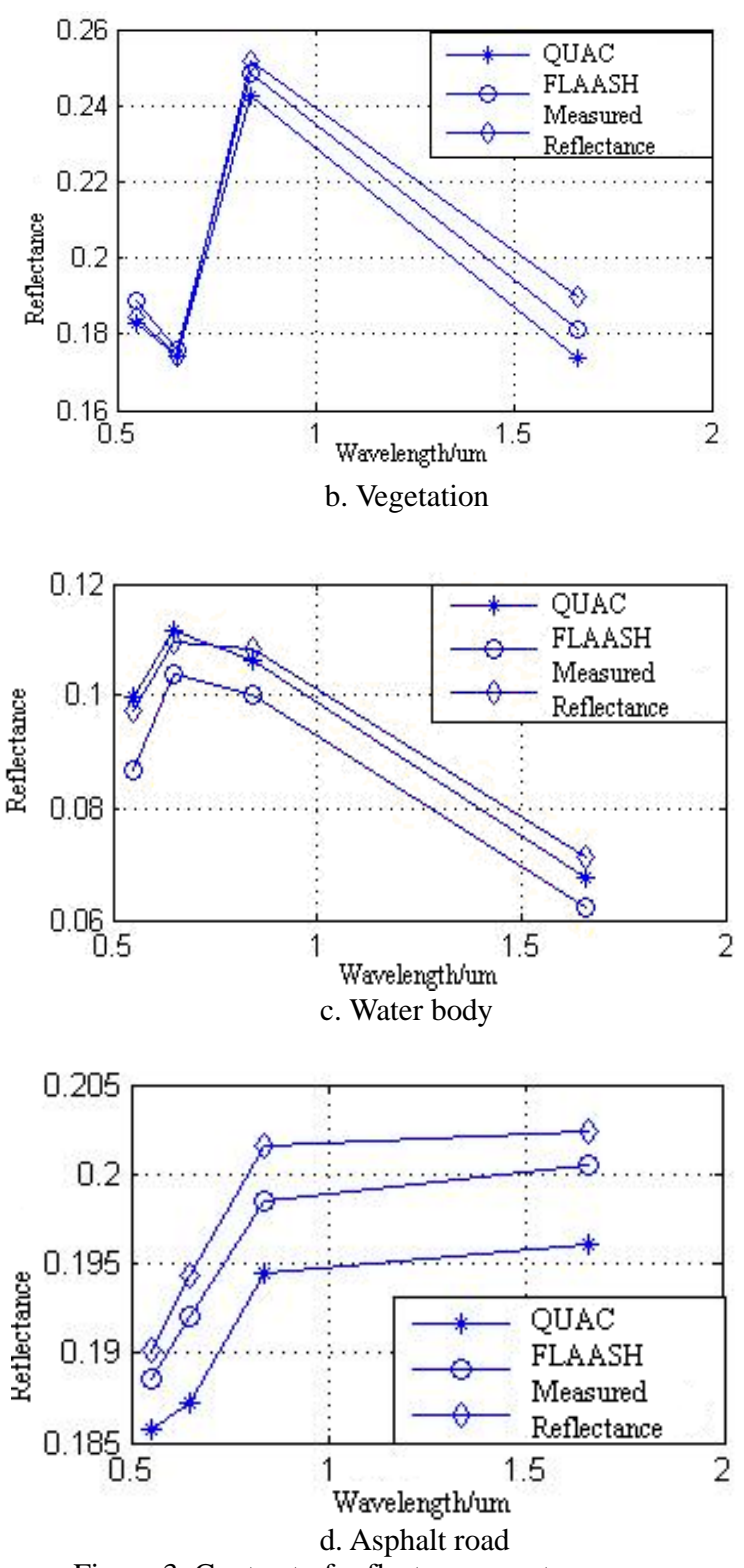

Figure 3. Contrast of reflectance spectrum curve

As the fourth band of SPOT-5 is beyond the band scope of wild radiometer, in order to ensure the integrity and aesthetics of the comparison chart, during the resampling process of measured spectral reflectance curves, the reflectance of the part (the fourth band) beyond the band scope shall be given man-made based on past experience and trends of the curve, thus comparative analysis mainly focuses on comparing the differences of the first three bands. We can conclude from the figure that the measured reflectance curve of the first three bands is more similar to the curve after the Model FLAASH atmospheric correction.

Soil reflectance is related to soil types, water content and surface roughness and other factors, and there are no obvious peaks and valleys of reflectance of soil surface under the natural state. The farmlands in the study region have just been harvested with straws covering the soil surface, so there is a valley at the 0.65 um of the three curves, similar to vegetation. However, it is not so different from the near-infrared reflectance. As shown in Figure 3(b), the vegetation spectral begin to decrease from the green band ( $0.55 \mathrm{um})$, a small valley at the red band $(0.65 \mathrm{um})$, which is due to the strong absorption effect of chlorophyll to red light and strong reflect action of chlorophyll to green light; then there is a peak of reflection at the rear-infrared $(0.84 \mathrm{um})$, which is subject to the effect of the structure of vegetation leaf cells, the unique characteristic of vegetation. Under the case that the reflectance of both models are slightly smaller than the measured reflectance, accuracy of FLAASH is higher than that of QUAC. The water body in the study area includes sediment, chlorophyll and other substances, and the is relatively shallow, also influenced by the bottom materials and the spectral transmittance of the water, so at the visible-near infrared, reflectance rises with the increase of wavelength instead of reducing. Reflectance curve of asphalt road surrounding the farmland rises slowly at the visible-near infrared band, and then becomes gentle after that. From Figure 3(d)we can see that the value of the measured reflectance curve is larger, and the reflectance after FLAASH atmospheric correction is more similar to the characteristics of the measured spectral.

3.3.3 RVI comparative analysis: The vegetation index (VI) is the index mainly reflecting the differences of vegetation among visible light, near infrared reflectance and soil background. In the field of remote sensing application, the index of various vegetations in certain conditions can be used for illustrating the growth situation of vegetation quantitatively. In order to evaluate the influences of the types of atmospheric corrective models on calculation of vegetation index, here the RVI is taken as the example; the three types of RVI value including the original DN value, the value of FLAASH and QUAC after atmospheric correction have been compared with each other.

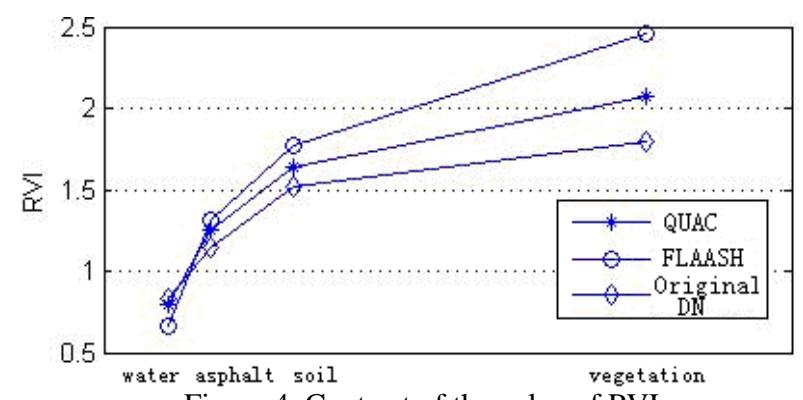

Figure 4. Contrast of the value of RVI

As shown in figure 4, for various ground features, the value of RVI after atmospheric correction is a little bit higher (excluding waters) than the calculation of original value of DN. Therein, the value of RVI after atmospheric correction increases obviously in the filed of vegetation, and the value of RVI after atmospheric correction based on the model of FLAASH nearly reaches 2.5. Thus it can be seen that the atmospheric correction could conspicuously increase the differences of RVI between the vegetation-covered area and non-vegetation covered area, which makes the vegetation information gets more prominent.

\section{CONCLUSIONS}

This paper makes a summary and comparison between Model FLAASH atmospheric correction and Model QUAC atmospheric correction, and on this basis the contrastive analysis of visual effect, reflectance spectral curve effect and RVI effect. The results of the trail show that: the images after the two model atmospheric corrections appear to be brighter visually and contrast is enhanced; while the reflection spectral characteristics of the surface features are largely restored and emphasis the vegetation information, indicating that the two models can be applied to the SPOT-5 images in southern hilly 
area. Model FLAASH reduces the effect effectively which the atmosphere has on the remote sensing image to enhance the image information with better accuracy than Model QUAC, but it depends on the input parameters and the calibration accuracy of instrument, so it applies to the cases that the calibration accuracy of instrument has reached the requirements and various atmospheric parameters have been known. Although the accuracy of Model QUAC is less than Model FLAASH, it depends less on the input parameters and the accuracy of instrument calibration with its specific applicability. For example, under the case that change detection is needed for multi-view images, fast atmospheric correction can be made first on the two images. Such simple and efficient input set can help finish the atmospheric correction task of "from figure to figure". It is certain that the two models also need further improvement, and enhancing the inversion accuracy in the area with complex terrain and under the complex atmospheric conditions is a valuable research direction.

\section{REFERENCES}

Chen Shupeng etc, Study of Information Mechanism of Remote Sensing. Science Press. 1998.

Zhao Yingshi etc, Applications of Remote Sensing Principles and Methods. Science Press. 2000.

Cheng Wei, Wang Liming, Tian Jiuqing. A Method of Atmospheric Correction Based on Shadow-pixel for Optical Satellite Data, Journal of Surveying and Mapping, 37(4). 2008.

M.W. Matthew, S. M. Adler-Golden, A. Berk, G. Felde, G. P. Anderson, D. Gorodetzky, S. Paswaters, and M. Shippert, "Atmospheric correction of spectral imagery: Evaluation of the FLAASH algorithm with AVIRIS data," in Proc. SPIE Conf. Algorithms and Technologies for Multispectral, Hyperspectral, and Ultraspectral Imagery IX, 2003.

Song Xiaoyu, Wang Jihua, Liu Liangyun, Huang Wenjiang, Zhao Chunjiang. Atmospheric Correction of Hyper-spectral Image: Evaluation of the FLAASH Algorithm with AVRIS Data. Remote Sensing Technology and Application.2005: 20 (4)

B.-C.Gao, M. J. Montes, C. O. Davis, and F.H. Goetz, "Atmospheric correction algorithms for hyperspectral remote sensing data of land and ocean", Remote Sensing of Environment, 113, pp.17-24, 2009.

Yang Jiaojun, Chen Yushi, Zhang Ye. Effect on Atmospheric Correction by Inputting Parameters of Model . Remote Sensing Application. 2008, June.

Berk, A., G.P. Anderson, P.K. Acharya, L.S. Bernstein, L. Muratov, J. Lee, M. Fox, S.M. Adler-Golden, J.H. Chetwynd, Jr., M.L. Hoke, R.B. Lockwood, J.A. Gardner, T.W. Cooley, C.C. Borel, P.E. Lewis and E.P. Shettle. "MODTRAN5:2006 Update," In Algorithms and Technologies for Multispectral, Hyperspectral, and Ultraspectral Imagery XII, Sylvia S. Chen, Paul E. Lewis, Editors, Proceedings of SPIE Vol. 6233, 2006.

Yang Hang, Zhang Xia, Shuai Tong, Tong Qingxi. 2010. Comparison between FLAASH Method and Empirical Linear Method of OMIS- II Image Atmospheric Correction. Bulletin of Surveying and Mapping. 2010, August.

ITT Visual Information Solutions (ITT VIS), “ENVI User's Guide, Version 4.8”, ITT Visual Information Solutions (ITT
VIS), Boulder (CO), USA.2010.

Wu Bin, Miao Fang, Ye Chengming, Huang Shuhanmao, Bi Xiaojia.Atmospheric Correction of Hyperspectral Remote Sensing Image Based on FLAASH. Computing Techniques for Geophysical and Geochemical Exploration. 2010,July

S.M. Adler-Golden, A. Berk, L.S. Bernstein, S. Richtsmeier, P.K. Acharya, M.W. Matthew, G.P. Anderson, C. Allred, L. Jeong, and J. Chetwynd, "FLAASH, A MODTRAN4 Atmospheric Correction Package for Hyperspectral Data Retrievals and Simulations," Proc. 7th Ann. JPL Airborne Earth Science Workshop, Pasadena, Calif., JPL Publication 97-21, pp. 9-14, 1998.

LIANG Shun-lin, FANG Hong-liang, CHEN Ming-zhen. Atmospheric Correction of Landsat ETM+ Land Surface Imagery-Part I: Methods. IEEE Transactions on Geo-science \& Remote Sensing, 39(11):2490-2498, 2001.

Perkins, T., S. Adler-Golden, M. Matthew, A. Berk, G. Anderson, J. Gardner and G. Felde, "Retrieval of Atmospheric Properties from Hyper and Multispectral Imagery with the FLAASH Atmospheric Correction Algorithm," In Remote Sensing of Clouds and the Atmosphere X, Klaus Schäfer; Adolfo T. Comerón; James R. Slusser; Richard H. Picard; Michel R. Carleer; Nicolaos Sifakis, Editors, Proceedings of SPIE Vol. 5979, 2005.

Hao Jianting, Yang Wunian, Li Yuxia, Hao Jianyuan. Atmospheric Correction of Hyperspectral Remote Sensing Image Based on FLAASH. Remote Sensing Application, 2008, January.

Wang Jihua etc. An Algorithm Based on 6S Model Removing Atmospheric Effects from Satellite Imaginery Pixel pixel by pixel. Optical Technique. 33(1): 11-15, 2007.

Guo Yunkai, Zhang Qisen. Research on Methods of Computer Classification of Generalized Angle-based Remote Sensing Image. China Journal of Highway and Transport. 15 (2), 2005.

Liu Yan, Wang Hong, Zhang Pu, Li Yang. Atmospheric Correction of Landsat TM Imagery by Using Meteorological Records. Remote Sensing for Land \& Resources. 388 (1), 2011.

Luo Cailian, Chen Jie, Le Tongchao. Atmospheric Correction on Landsat ETM+ Satellite Image Based on FLAASH Model. Protection Forest Science and Technology. 86 (5), 2008. 